\title{
PERSISTENCE AND BIFURCATION ANALYSIS ON A PREDATOR-PREY SYSTEM OF HOLLING TYPE
}

\author{
Debasis MukherJeE ${ }^{1}$
}

\begin{abstract}
We present a Gause type predator-prey model incorporating delay due to response of prey population growth to density and gestation. The functional response of predator is assumed to be of Holling type II. In absence of prey, predator has a density dependent death rate. Sufficient criterion for uniform persistence is derived. Conditions are found out for which system undergoes a Hopf-bifurcation.
\end{abstract}

Mathematics Subject Classification. 34D23, 34D45, 92D25.

Received: January 22, 2002.

\section{INTRODUCTION}

The dynamic relationship between predators and their prey create major interest to many investigators due to universal existence and importance [3]. These problems looks simple as it stands, sometimes it becomes quite complicated to answer the question of survival and oscillation. These theory made a rapid progress in the last few years, still some problems remain unsolved (Berryman [3], Kuang and Freedman [14], Kuang [9-12], Beretta and Kuang [2]).

It is widely known that past history as well as present conditions can influence population dynamics and such interactions has motivated the introduction of delays in population growth models. Sometimes delay can change the dynamics. In delay models with complicated dynamics, the question of persistence (long term survival of the population) and stability switching are important. Modelling of interacting species with time delay is being dealt recently by Kuang [13], Freedman and Rao [7], Cao and Freedman [4], Mukherjee and Roy [16]. Cao and Freedman [4] considered a general class of models of prey-predator interactions with time delay due to gestation only. They derived criteria for uniform persistence and global attractivity of a positive equilibrium. Wang and Ma [20] derived conditions for uniform persistence for Lotka-Volterra prey-predator system with finite number of discrete delays. Farkas and Freedman [6] obtained criteria for global stability of general model of two-predator-one prey system in terms of restrictions on the growth functions. The conditions seem to be difficult to check. Mukherjee and Roy [16] obtained sufficient conditions for uniform persistence and global stability of interior equilibrium of a delayed ecological model involving a resource and two predators.

Keywords and phrases. Persistance, bifurcation, stability, holling type II.

1 Department of Mathematics, Vivekananda College, Thakurpukur, Kolkata 700063, India.

e-mail: debasis_mukherjee2000@yahoo.co.in 
In general it has been found that incorporating time delays is a destabilizing process, and that increasing the lag could cause a stable equilibrium to become unstable and/or cause populations to oscillate (see May [15], Taylor and Sokal [18], Veilleux [19], Adams et al. [1]).

In this paper we are interested to study the effect of delays on prey-predator system with Holling type II functional response. This type of situation is investigated by Dale et al. [5]. In [5], Dale et al. studied the predatory behaviour of Wolves (Canis lupus) in Gates of Arctic National Park and Preserve in the Central Brookes Range of Alaska, a wilderness area inside the Arctic Circle. Wolves prey primarily on Caribou (Rangifer tarandas), although they will also take Dall sheep (Ovics dalli) and moose (Alces alces). Dale and coworkers estimated the functional response of wolves to caribou abundance, which varies substantially, by four wolf packs for a period of 30 days in the winter of 1990 and counting the number of kills per wolf are the number of caribou available on each day. When the number of kills per wolf per day was plotted against the estimated number of caribou per $\mathrm{km}^{2}$, the data fit the Holling type II functional response curve. For details see Ricklefs and Miller [17]. We utilize two types of delays in our model. One for the growth equation of prey and another for gestation. We address two questions in this paper (1) under what conditions the populations will survive in long run (ii) it is already known that single delay in the growth equation of prey lead oscillation, will this behaviour will be reflected for two delay terms also?

The paper is organized as follows. In Section 2, we present our model and discuss the boundedness of solutions and uniform persistence. Bifurcation analysis is given in Section 3. A brief discussion concludes in Section 4.

\section{THE MODEL}

We consider the response of prey population growth to density is time-delayed, that is, when effect of density dependence reflects the density of population $\tau_{1}$ time units in the past. Another time delay $\tau_{2}$ is taken for gestation. The model is described by the following:

$$
\begin{aligned}
& \frac{\mathrm{d} x}{\mathrm{~d} t}=x(t)\left[1-\frac{x\left(t-\tau_{1}\right)}{k}-\frac{y}{1+x}\right] \\
& \frac{\mathrm{d} y}{\mathrm{~d} t}=y(t)\left[-d+\frac{h x\left(t-\tau_{2}\right)}{1+x\left(t-\tau_{2}\right)}-f y\right]
\end{aligned}
$$

with initial conditions

$$
x(t)=x_{0}(t) \geq 0, \quad t \in[-\tau, 0], \quad \tau=\max \left(\tau_{1}, \tau_{2}\right), \quad y(0)=y_{0} \geq 0 .
$$

$x_{0}:[-\tau, 0] \rightarrow[0, \infty)$ is assumed to be continuous function.

Hence $x$ represents the density of the prey and $y$ that of predator. $k$ denotes the carrying capacity of environment. $d$ is the death rate of predator, $f$ is the intraspecific competition coefficient of the predator, $h$ is the conversion of biomass constant. All the parameters in system (1) are positive.

The following result shows that solutions of system (1) are bounded.

Theorem 1. Suppose $h k>d$. Then solutions of (1) are uniformly bounded.

Proof. From the first equation in (1), we have

$$
\lim _{t \rightarrow \infty} \sup x(t) \leq k \mathrm{e}^{\tau_{1}}=k_{1} \text { (say) }
$$

Again from the second equation in (1), we have

$$
\frac{\mathrm{d} y}{\mathrm{~d} t} \leq y(t)\left[-d+h k_{1}-f y\right] .
$$


Applying the same arguments as before, we have

$$
\lim _{t \rightarrow \infty} \sup y(t) \leq \frac{h k_{1}-d}{f} .
$$

This completes the proof.

Our next theorem shows that system (1) is uniformly persistent.

Theorem 2. Let $\frac{h k}{1+k}>d$. Then system (1) is uniformly persistent.

In order to prove Theorem 2, we require uniform persistence theory for infinite dimensional systems from [8]. Let $X$ be a complete metric space. Suppose that $X^{\circ}$ is open, dense in $X$ and $X^{\circ} \cup X_{\circ}=X, X_{\circ} \cap X^{\circ}=\phi$. Assume that $T(x)$ is a $C^{\circ}$ semigroup on $X$ satisfying

$$
\begin{aligned}
& T(t): X^{\circ} \rightarrow X^{\circ} \\
& T(t): X_{\circ} \rightarrow X_{\circ} .
\end{aligned}
$$

Let $T_{b}(t)=\left.T(t)\right|_{X_{\circ}}$ and let $A_{\mathrm{b}}$ be the global attractor for $T_{\mathrm{b}}(t)$.

Lemma 1. Suppose that $T(t)$ satisfies $(*)$ and we have the following:

(i) there is a $t_{0} \geq 0$ such that $T(t)$ is compact for $t \geq t_{0}$;

(ii) $T(t)$ is point dissipative in $X$;

(iii) $\bar{A}_{\mathrm{b}}=\cup_{x \in A_{\mathrm{b}}}$ is isolated and has an acyclic covering $\bar{M}$ where

$$
\bar{M}=\left\{M_{1}, M_{2}, \ldots, M_{n}\right\}
$$

(iv) $W^{s}\left(M_{i}\right) \cap X^{\circ}=\phi$ for $i=1,2, \ldots, n$.

Then $X_{o}$ is a uniform repellor with respect to $X^{\circ}$, i.e., there is an $\in>0$ such that for any $x \in X^{\circ}, \lim _{t \rightarrow \infty} \inf d(T(t) x$, $\left.X_{\circ}\right) \geq \epsilon$, where $d$ is the distance of $T(t) x$ from $X_{\circ}$.

We are now able to state the Proof of Theorem 2.

Proof of Theorem 2. The condition in Theorem 2 implies that $h k>d$ and hence by Theorem 1 it follows that $T(t)$ is point dissipative. We know that $E_{0}(0,0)$ and $E_{1}(k, 0)$ are the only boundary equilibria of system (1). The origin is clearly unstable. We linearize around $E_{1}$ and obtain the characteristic equation.

$$
\left(\lambda-\frac{h k}{1+k}+d\right)\left(\lambda+\mathrm{e}^{\lambda \tau_{1}}\right)=0
$$

The eigenvalues of $\lambda+\mathrm{e}^{\lambda \tau_{1}}=0$ all have negative real parts. There is a unique positive eigenvalue $\lambda=\frac{h k}{1+k}-d$. Thus the stable set of $E_{1}$ does not intersect the positive cone. $E_{0}$ and $E_{1}$ are isolated invariant sets on the boundary. Since $E_{0}$ is unstable, $\bar{A}_{\mathrm{b}}$ is just the union of the two steady states. Taking $M_{i}$ to be these steady states, there are no cycles on the boundary. Therefore, the result follows by Lemma 1 .

Theorem 3. If $\frac{h k}{1+k}<d$ then $E_{1}$ is locally asymptotically stable.

Proof. Proof is obvious. 


\section{Bifurcation ANALYSIS}

In this section we derive criteria for Hopf-bifurcation. An interior rest point $E^{*}=\left(x^{*}, y^{*}\right)$ is determined by the following equations:

$$
\begin{gathered}
1-\frac{x^{*}}{k}-\frac{y^{*}}{1+x^{*}}=0, \\
-d+\frac{h x^{*}}{1+x^{*}}-f y^{*}=0 .
\end{gathered}
$$

The above two equations yield

$$
f x^{* 3}-f(k-2) x^{* 2}-(2 f k-f-h k+d h) x^{*}-k(f+d)=0 .
$$

Clearly equation (2) has one positive root which lies between 0 and $k$, if $\frac{h k}{1+k}>d$. We linearize system (1) around $E^{*}$ when $\tau_{1}=\tau_{2}=\tau$ and obtain the characteristic equation

$$
\lambda^{2}+a_{1} \lambda+a_{2} \lambda \mathrm{e}^{-\lambda \tau}+a_{3}+a_{4} \mathrm{e}^{-\lambda \tau}=0
$$

where

$$
\begin{aligned}
& a_{1}=y^{*}\left(f-\frac{x^{*}}{\left(1+x^{*}\right)^{2}}\right) \\
& a_{2}=\frac{x^{*}}{k} \\
& a_{3}=-\frac{f y^{* 2} x^{*}}{\left(1+x^{*}\right)^{2}} \\
& a_{4}=x^{*} y^{*}\left[\frac{h}{\left(1+x^{*}\right)^{3}}+\frac{f}{k}\right]
\end{aligned}
$$

Suppose $\lambda=i \sigma, \sigma>0$ is a root of (3) for some $\tau$.

Assume $a_{3}+a_{4} \neq 0$.

This assumption implies that $\sigma \neq 0$. We have

$$
\begin{gathered}
a_{3}-\sigma^{2}+a_{2} \sigma \sin \sigma \tau+a_{4} \cos \sigma \tau=0, \\
a_{1} \sigma+a_{2} \sigma \cos \sigma \tau-a_{4} \sin \sigma \tau=0 .
\end{gathered}
$$

Thus

$$
\left(\sigma^{2}-a_{3}\right)^{2}+a_{1}^{2} \sigma^{2}=a_{2}^{2} \sigma^{2}+a_{4}^{2}
$$

Hence

$$
\sigma^{4}+\left(a_{1}^{2}-a_{2}^{2}-2 a_{3}\right) \sigma^{2}+a_{3}^{2}-a_{4}^{2}=0
$$

Its roots are:

$$
\sigma_{ \pm}^{2}=\frac{1}{2}\left\{\left(a_{2}^{2}+2 a_{3}-a_{1}^{2}\right) \pm \sqrt{\left(a_{2}^{2}+2 a_{3}-a_{1}^{2}\right)^{2}-4\left(a_{3}^{2}-a_{4}^{2}\right)}\right\} .
$$

If $a_{3}^{2} \leq a_{4}^{2}$, then there is only one imaginary solution $\lambda=i \sigma_{+}, \sigma_{+}>0$. If $a_{3}^{2}>a_{4}^{2}$, there are two imaginary solutions $\lambda_{ \pm}=i \sigma_{ \pm}$with $\sigma_{+}>\sigma_{-}>0$, provided that the following are true:

(i) $a_{2}^{2}+2 a_{3}-a_{1}^{2}>0$ and (ii) $\left(a_{2}^{2}+2 a_{3}-a_{1}^{2}\right)^{2}>4\left(a_{3}^{2}-a_{4}^{2}\right)$ and no such solutions otherwise. 
Now,

$$
\begin{aligned}
\operatorname{sign}\left\{\frac{\mathrm{d}(\operatorname{Re} \lambda)}{\mathrm{d} \tau}\right\}_{\lambda=i \sigma} & =\operatorname{sign}\left\{\operatorname{Re}\left(\frac{\mathrm{d} \lambda}{\mathrm{d} \tau}\right)^{-1}\right\}_{\lambda=i \sigma} \\
& =\operatorname{sign}\left\{\operatorname{Re}\left[\frac{-\left(2 \lambda+a_{1}\right)}{\lambda\left(\lambda^{2}+a_{1} \lambda+a_{3}\right)}\right]_{\lambda=i \sigma}+\operatorname{Re}\left[\frac{a_{2}}{\lambda\left(a_{2} \lambda+a_{4}\right)}\right]_{\lambda=i \sigma}\right\} \\
& =\operatorname{sign}\left\{\frac{a_{1}^{2}-2\left(a_{3}-\sigma^{2}\right)}{\left(a_{3}-\sigma^{2}\right)^{2}+a_{1}^{2} \sigma^{2}}-\frac{a_{2}^{2}}{a_{4}^{2}+a_{2}^{2} \sigma^{2}}\right\} \\
& =\operatorname{sign}\left\{a_{1}^{2}-2 a_{3}-a_{2}^{2}+2 \sigma^{2}\right\}
\end{aligned}
$$

By inserting the expression for $\sigma_{ \pm}^{2}$, it is seen that the sign is positive for $\sigma_{+}^{2}$ and negative for $\sigma_{-}^{2}$. In the case of $a_{3}^{2}<a_{4}^{2}$, only one imaginary roof exists, $\lambda=i \sigma_{+}$; therefore, the only crossing of the imaginary axis is from left to right as $\tau$ increases and stability of the positive equilibrium can only be lost and not regained. In the case of $a_{3}^{2}>a_{4}^{2}$, crossing from left to right with increasing $\tau$ occurs whenever $\tau$ assumes a value corresponding to $\sigma_{+}$and crossing from right to left occurs for values of $\tau$ corresponding to $\sigma_{-}$. From equations (4) and (5), we obtain the following two sets of values of $\tau$ for which there are imaginary roots:

$$
\tau_{n, 1}=\frac{\theta_{1}}{\sigma_{+}}+\frac{2 n \pi}{\sigma_{+}}
$$

where $0 \leq \theta_{1}<2 \pi$ and

$$
\begin{aligned}
\cos \theta_{1} & =\frac{a_{1} a_{2} \sigma_{+}^{2}+a_{4}\left(a_{3}-\sigma_{+}^{2}\right)}{\sigma_{+}^{2} a_{2}^{2}+a_{4}^{2}} \\
\sin \theta_{1} & =\frac{a_{4} a_{1} \sigma_{+}-a_{2} \sigma_{+}\left(a_{3}-\sigma_{+}^{2}\right)}{a_{2}^{2} \sigma_{+}^{2}+a_{4}^{2}}
\end{aligned}
$$

and

$$
\tau_{n, 2}=\frac{\theta_{2}}{\sigma_{-}}+\frac{2 n \pi}{\sigma_{-}}
$$

where $0 \leq \theta_{2}<2 \pi$ and

$$
\begin{aligned}
\cos \theta_{2} & =\frac{a_{1} a_{2} \sigma_{-}^{2}+a_{3}\left(a_{3}-\sigma_{-}^{2}\right) a_{4}}{a_{2}^{2} \sigma_{-}^{2}+a_{4}^{2}} \\
\sin \theta_{2} & =\frac{a_{4} a_{1} \sigma_{-}-a_{2} \sigma_{-}\left(a_{3}-\sigma_{-}^{2}\right)}{a_{2}^{2} \sigma_{-}^{2}+a_{4}^{2}}
\end{aligned}
$$

where $n=0,1,2, \ldots$

In the case that $a_{3}^{2}<a_{4}^{2}$, only $\tau_{0,1}$ need be considered, since if $E^{*}$ is asymptotically stable for $\tau=0$, then it remains asymptotically stable until $\tau_{0,1}$ and it is unstable thereafter. At the value of $\tau=\tau_{0,1}$, (3) has pure imaginary roots, $\pm \sigma_{+}$. In the case that $a_{3}^{2}>a_{4}^{2}$, if $E^{*}$ is table for $\tau=0$, then it must follow that $\tau_{0,1}<\tau_{0,2}$, since multiplicity of roots with positive real parts cannot become negative. We note that

$$
\tau_{n+1,1}-\tau_{n, 1}=\frac{2 \pi}{\sigma_{+}}<\frac{2 \pi}{\sigma_{-}}=\tau_{n+1,2}-\tau_{n, 2} .
$$

Therefore, there can be only a finite number of switches between stability and instability.

Thus we have the following theorem. 
Theorem 4. If $a_{3}+a_{4}>0$ then the unstable equilibrium of (1) becomes stable for any $\tau \geq 0$. If $E^{*}$ is asymptotically stable for $\tau=0$, then it is uniformly asymptotically stable for $\tau<\tau_{0,1}$ and it becomes unstable for $\tau>\tau_{0,1}$.

If (i) $a_{3}+a_{4}<0$, (ii) $a_{2}^{2}+2 a_{3}-a_{1}^{2}>0$ and (iii) $\left(a_{2}^{2}+2 a_{3}-a_{1}^{2}\right)>4\left(a_{3}^{2}-a_{4}^{2}\right)$ then the stability of $E^{*}$ can change a finite number of times at most as $\tau$ is increased, and eventually it becomes unstable.

The condition $a_{3}+a_{4}>0$ can be written in the form

$$
f x^{* 2}+3 f x^{* 2}+(3 f+k d-k h) x^{*}+h k+f+k d>0 .
$$

If the conversion of biomass constant remains below a certain threshold value then above condition is satisfied. Similarly conditions $a_{3}+a_{4}<0$ requires that conversion of biomass constant exceeds sufficiently a certain threshold value.

\section{Discussion}

In this paper we have considered a prey-predator model of Holling type functional response. We have incorporated delays on prey growth response and in predator intake rate. It has been shown that the system is uniformly bounded as long as carrying capacity of the environment exceeds a certain threshold value. For long term survival of the populations, the death rate of predator must be below a certain threshold value. Clearly the result shows that delay is harmless for persistence. Theorem 4 shows that if conversion of biomass constant remains below a certain threshold value then delay has stabilizing effect on the system. If delay is increased then stability of the equilibrium is lost. Again if conversion of biomass constant exceeds a certain threshold value then stability switches finite number of times.

\section{REFERENCES}

[1] V.D. Adams, D.L. DeAngelis and R.A. Goldstein, Stability analysis of the time delay in a Host-Parasitoid Model. J. Theoret. Biol. 83 (1980) 43-62.

[2] E. Beretta and Y. Kuang, Convergence results in a well known delayed predator-prey system. J. Math. Anal. Appl. 204 (1996) $840-853$.

[3] A.A. Berryman, The origins and evolution of predator-prey theory. Ecology 73 (1992) 1530-1535.

[4] Y. Cao and H.I. Freedman, Global attractivity in time delayed predator-prey system. J. Austral. Math. Soc. Ser. B. 38 (1996) 149-270.

[5] B.W. Dale, L.G. Adams and R.T. Bowyer, Functional response of wolves preying on barren ground caribou in a multiple prey ecosystem. J. Anim. Ecology 63 (1994) 644-652.

[6] M. Farkas and H.I. Freedman, The stable coexistence of competing species on a renewable resource. 138 (1989) $461-472$.

[7] H.I. Freedman and V.S.H. Rao, The trade-off between mutual interface and time lags in predator-prey systems. Bull. Math. Biol. 45 (1983) 991-1004.

[8] J.K. Hale and P. Waltman, Persistence in infinite dimensional systems. SIAM J. Math. Anal. 20 (1989) 388-395.

[9] Y. Kuang, Non uniqueness of limit cycles of Gause type predator-prey systems. Appl. Anal. 29 (1988) 269-287.

[10] Y. Kuang, On the location and period of limit cycles in Gause type predator-prey systems. J. Math. Anal. Appl. 142 (1989) 130-143.

[11] Y. Kuang, Limit cycles in a chemostat related model. SIAM J. Appl. Math. 49 (1989) 1759-1767.

[12] Y. Kuang, Global stability of Gause type predator-prey systems. J. Math. Biol. 28 (1990) 463-474.

[13] Y. Kuang, Delay Differential Equations with Applications in Population Dynamics. Academic Press, San Diego (1993).

[14] Y. Kuang and H.I. Freedman, Uniqueness of limit cycles in Gause type predator-prey systems. Math. Biosci. 88 (1988) 67-84.

[15] R.M. May, Time-delay versus stability in population models with two and three trophic levels. Ecology 54 (1973) $315-325$.

[16] D. Mukherjee and A.B. Roy, Uniform persistence and global attractivity theorem for generalized prey-predator system with time delay. Nonlinear Anal. 38 (1999) 59-74.

[17] R.E. Ricklefs and G.L. Miller, Ecology. W.H. Freeman and Company, New York (2000).

[18] C.E. Taylor and R.R. Sokal, Oscillations of housefly population sizes due to time lags. Ecology 57 (1976) 1060-1067.

[19] B.G. Vielleux, An analysis of the predatory interactions between Paramecium and Didinium, J. Anim. Ecol. 48 (1979) $787-803$.

[20] W.D. Wang and Z.E. Ma, Harmless delays for uniform persistence. J. Math. Anal. Appl. 158 (1991) 256-268. 